\title{
Research on Credit Risk Evaluation for Small and Medium-sized Enterprises in Supply Chain Based on BP Neural Network
}

\author{
Xiaoping Zhang ${ }^{\mathrm{a}}$, Chen Chen ${ }^{\mathrm{b}}$ \\ Department of Accounting, Hebei Financial University,Baoding, China

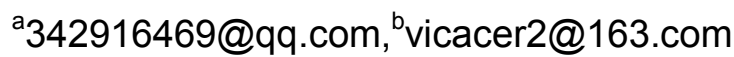

\begin{abstract}
Keywords: Supply chain finance; BP neural network; Small and medium-sized enterprises; Credit risk assessment
\end{abstract}

\begin{abstract}
Abstract More attention to supply chain finance as a new form of financing channel is paid with the increasing highlight of the financing difficulty of small and medium-sized enterprises (SMEs). It is crucial for scientific and accurate risk assessment to control the control of the credit risk of supply chain finance. BP neural network model is used to open up a new way for credit risk assessment of SMEs based on the establishment and improvement of an index system of credit risk evaluation for SMEs. BP neural network model is a smart algorithm with self-learning and adaptive characteristics. The BP artificial neural network model was analyzed and detected in a practical case. The feasibility of the model was proved. The result is satisfactory. The model can be used to assess SMEs' credit risk in the supply chain.
\end{abstract}

\section{Introduction}

Small medium enterprise financing is a long-standing problem. In recent years, the rapid development of the supply chain finance in the past two years to a certain extent, ease the small medium enterprise financing difficulties. At present, the expert of supply chain finance (SCF) are defined as follows: based on the grasp of the supply chain transaction details, and the characteristics of the structure, with the help of core enterprise credit strength or single transaction self-liquidating degree and circulation of goods value, supply chain finance provides a comprehensive range of financial services for a single or multiple enterprises. Because the proportion accounted for the small medium enterprise financing lease business in the bank is very small, fledgling plus supply chain financing mode, there is no established for supply chain in the small medium enterprise credit risk evaluation index system[1]. In order to be able to timely, dynamically and accurately evaluating and monitoring the risk of small medium enterprise financing process, this paper established a comprehensive index system for small medium enterprise credit risk evaluation, establish a benign development between enterprises, banks, and supply chain of industrial ecosystem. The traditional risk assessment methods include principal component analysis (PCA), analytic hierarchy process (AHP) and fuzzy mathematics method, etc. But the above methods have the following deficiencies: the weight of evaluation are easily influenced by subjective factors, so the decision may be easy distortion. To solve this problem, this paper adopts a risk assessment method based on BP neural network which has the characteristics of self-learning and adaptive ability[2].

\section{BP neural network}

Neural network is essentially a simulation system for the human brain's thinking process. Its core is the mathematical models and algorithms, and the physical implementation is computer software. Just like thins are composed of atoms, neural network is composed of many interconnected neurons[3]. Fig. 1 shows the basic work principle of neurons, and the mathematical expression is as follows:

$$
y=f\left(\sum w_{i} x_{i}\right)
$$


Where $x_{i}$ and $y$ are respectively the input and output. $w_{i}$ is weight coefficient. $f$ is characteristic function, which reflects the mapping relationship between inputs and outputs, and it is usually a nonlinear function.

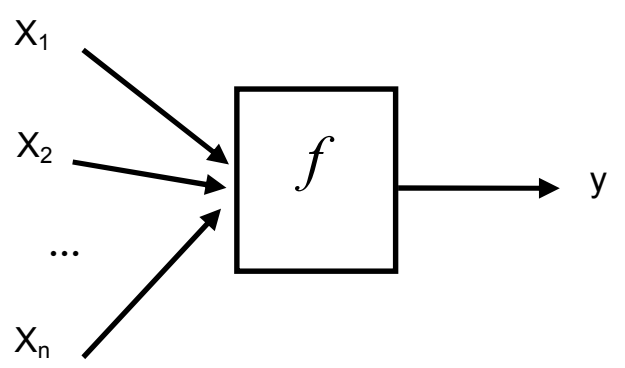

Fig. 1 The neuron model

This seemingly simple model will produce a powerful neural network combing different network topology and network algorithms together. Neural network consists of input layer, hidden layer and output layer, which is shown in Fig. 2.

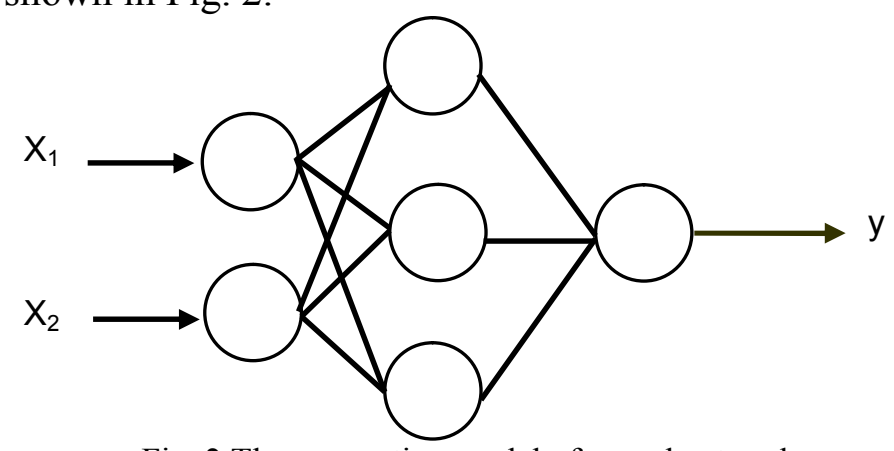

Fig. 2 The connection model of neural network

With a lot of development of neural networks, it was found that no matter how the organizational structure of the network it is, It is always has the following two characteristics:

(1) Self-learning

Neural networks can be modified according to the external environment of their own behavior in order to adapt to the external environment, which is mainly due to its learning process. Learning is often the first step in using neural networks. When a group of information is input, neural networks can continue to adjust its internal parameters (or say weighting coefficient), and eventually produce a series of consistent output.

(2) Generation

Once after the self-learning, the response of neural network, to some extent, to the reducing of input information and their own local defects are no longer sensitive. This mechanism can make the neural network has a strong fault tolerance and reduce the input data quality requirements.

BP neural network is a multilayer forward network based on back-propagation errors[4]. The network structure is consist of nodes and arrows line. Nodes simulate the human brain 's neurons, and arrows line simulate the connected relationship between neurons. According to different neurons location, the network can be divided into three layers, namely input layer, hidden layer and output layer. Nodes in input layer are responsible for data input, and themselves have no computing function. Nodes in the hidden layer and output layer have a increasing and a activation function. Information processing mechanism on BP neural network is made up of nodes increases and the activation functions.

The increasing function means that the multiplications of a node's all input and its corresponding connection are all added up. For example, the increasing function value of the first node in hidden layer is defined as below.

$$
x_{1}=\sum_{i} D_{i} w_{i 1} \text {. }
$$


Where $x_{1}$ is the input value of the first node in hidden layer. $D_{i}$ is the input data of the $i$ th node in input layer. $w_{i 1}$ is the weight value from the $i$ th node in input layer to the first node in hidden layer.

The activation function means that the added value mentioned above function are transformed to the output value of the node through a certain mathematical method. For example, the output value of the first node in hidden layer is defined as below.

$$
y_{1}=g\left(x_{1}\right) \text {. }
$$

Where $g(x)$ is named as activation function. The common activation functions include Sigmoid function, proportional function, saturation function, hyperbolic functions and so on.

\section{The credit risk evaluation model based on BP neural network}

In the past, the evaluation of banks' financing objects are mainly single enterprise, and the assessment are required according to the static financial data. Thus, there exists many problems, because the financial data of small and medium-sized enterprises are scattered and not specification, and the transparency is relatively low, which make it harder for banks to carry on the scientific evaluation[1]. Financial point of view of the supply chain thought is based on the correlation between industry supply chain, assess to the enterprise supply chain operation, in order to dynamically assess the real risks of credit project. Supply chain financial interests of relevant subjects include: the supply chain core, the small medium enterprises of the core enterprise supply chain upstream and downstream, loan banks, and related logistics enterprises. According to the index design principles and methods, on the basis of a large number of literature research and expert investigation, the credit risk evaluation index system of small and medium-sized enterprises under the financial perspective of supply chain was established, which contains four major categories and 21 indicators, as shown in Table 1.

Table 1. The credit risk evaluation index system of small and medium-sized enterprises

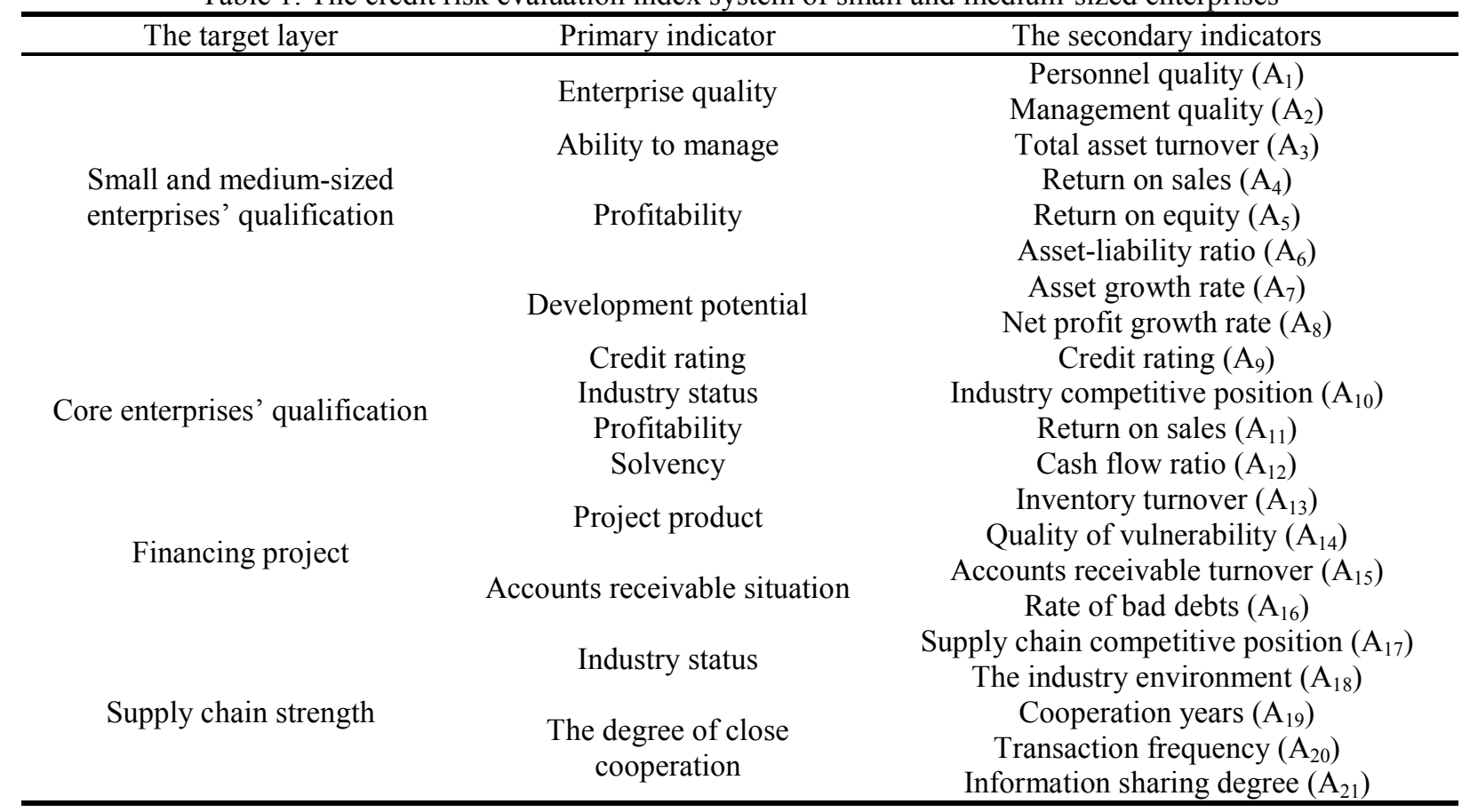

The key of BP neural network is to determine the number of network hierarchy and the number of neurons in every level. Some research shows that the learning of BP network with 4 layered structure is better..Therefore, this paper uses the 4 level BP network structure to train, the 4 level includes one input data layer, two intermediate hidden layers and one output data layer. 


\section{Case study}

From the RESSET financial database, 20 groups data were selected which have the obvious features of supply chain, and were transformed into Table 2. Sample data involves building materials, transportation, iron and steel industry, biotechnology, pharmaceutical and other industries of small and medium-sized enterprises. Through the simulation of Matlab software, the information of 1-15 group are selected as the training sample of the model, and the sample of 15-19 group are used as the test sample. The twentieth group of data is the object to be evaluated.. The learning rate and error are respectively set as 0.05 and 0.01 .

Put test sample data into the model, the results are shown in Table 2. The results show that, compared with the experts expected results, the results of the model computation has a high consistency, the final error is only 0.0058, which is within the set range and satisfies the requirements. Therefore, this model is practical and scientific.

Table 2. Sample data

\begin{tabular}{cccccc}
\hline No. & $\mathrm{A}_{1}$ & $\mathrm{~A}_{2}$ & $\cdots$ & $\mathrm{A}_{20}$ & $\mathrm{~A}_{21}$ \\
\hline 1 & 0.6 & 0.6 & & 0.9 & 0.6 \\
2 & 0.9 & 0.6 & & 0.9 & 0.6 \\
3 & 0.3 & 0.6 & & 0.3 & 0.3 \\
4 & 0.6 & 0.6 & & 0.6 & 0.9 \\
5 & 0.6 & 0.3 & & 0.6 & 0.6 \\
6 & 0.6 & 0.6 & & 0.9 & 0.6 \\
7 & 0.3 & 0.6 & & 0.9 & 0.3 \\
8 & 0.6 & 0.6 & & 0.6 & 0.3 \\
9 & 0.3 & 0.6 & & 0.9 & 0.3 \\
10 & 0.9 & 0.9 & $\cdots$ & 0.9 & 0.6 \\
11 & 0.6 & 0.6 & & 0.6 & 0.6 \\
12 & 0.6 & 0.6 & & 0.9 & 0.6 \\
13 & 0.9 & 0.9 & & 0.9 & 0.9 \\
14 & 0.9 & 0.6 & & 0.6 & 0.6 \\
15 & 0.6 & 0.6 & & 0.3 & 0.3 \\
16 & 0.9 & 0.9 & & 0.9 & 0.6 \\
17 & 0.9 & 0.6 & & 0.3 & 0.6 \\
18 & 0.9 & 0.9 & & 0.9 & 0.3 \\
19 & 0.6 & 0.6 & & 0.3 & 0.6 \\
20 & 0.6 & 0.6 & &
\end{tabular}

Apply the proposed model to evaluate the final set of data, the data for the fifth column data are shown in Table 3, which proved that the small and medium-sized enterprise credit risk rating is general, which needs to strengthen risk control.

Table 3. The results comparison

\begin{tabular}{cccccc}
\hline No. & 1 & 2 & 3 & 4 & 5 \\
\hline Evaluation results & 0.72 & 0.52 & 0.83 & 0.56 & 0.55 \\
Expected results & 0.7360 & 0.5157 & 0.8396 & 0.5875 & 0.5573 \\
\hline
\end{tabular}

\section{Conclusions}

The rapid development of supply chain finance in the past two years to a certain extent, alleviate the financing difficulties of small and medium-sized enterprise. But due to not have a complete system of credit risk and scientific risk evaluation method, affected by the previous risk assessment model at the same time, in the small medium-sized enterprise credit assessment, small medium-sized enterprise credit level is low, which largely limits the small medium-sized enterprise financing. In order to effectively control the risk of bank, at the same time truly broaden the small medium-sized enterprise financing channels, achieve a win-win situation, this paper establish a comprehensive credit risk evaluation system, and adopts BP neural network to evaluate the risk of small enterprises. The results is satisfied, indicate that the proposed model can be used in practical application. 


\section{References}

[1] Ladislav Mura, Ján Buleca. Evaluation of Financing Possibilities of Small and Medium Industrial Enterprises. Procedia Economics and Finance. Vol. 3 (2012), p. 217-222.

[2] Samuel B. Moore, Susan L. Manring. Strategy development in small and medium sized enterprises for sustainability and increased value creation. Journal of Cleaner Production. Vol. 17, Issue 2 (2009), p. 276-282.

[3] Hu Junguo, Zhou Guomo, Xu Xiaojun. Using an improved back propagation neural network to study spatial distribution of sunshine illumination from sensor network data. Ecological Modelling. Volume 266, Issue 24(2013): 86-96.

[4] Xuefeng Zhao, Qin Ba, Lei Zhou, Weijie Li, Jinping Ou. BP neural network recognition algorithm for scour monitoring of subsea pipelines based on active thermometry. International Journal for Light and Electron Optics. Volume 125, Issue 18( 2014), p. 5426-5431. 